\title{
MaxElide and the Re-binding Problem
}

\author{
Shoichi Takahashi \& Danny Fox \\ MIT
}

\section{Introduction}

It is well-known that ellipsis constructions involving bound variables are sometimes ambiguous. For instance, the sentence uttered by Speaker B in (1) can be understood either as a statement about John (Speaker B 1, strict identity) or as a statement about Bill alone (Speaker B2, sloppy identity). (Angle brackets are used to mark the elided constituent (EC) and focused material is written in capital letters.)

(1) Speaker A: John admires his professor.

Speaker B: BILL also does < admire his professor>.

1. Bill admires John's professor.

(strict identity)

2. Bill admires Bill's professor.

(sloppy identity)

One might suggest that sloppy identity results from a configuration in which a variable is free inside EC, and is bound by an antecedent outside EC, as illustrated in (2).

(2) John [vP admires his professor]. Bill also does $<_{\mathrm{EC}}$ admire his professor $>$.

Such an analysis, however, raises questions about the semantic licensing conditions on ellipsis (henceforth, the parallelism condition). Suppose we adopt the rather simple condition proposed by Sag (1976) and Williams (1977), namely, that ellipsis of EC requires semantic identity with an antecedent constituent (AC). If sloppy identity involves the representation in (2), it is not obvious that the parallelism condition is met. ${ }^{1}$

For this and other reasons (to which we return), Sag and Williams argue that there is an alternative analysis of sloppy identity. They claim that the relevant variable is not free within EC in sloppy identity, but is instead bound by a $\lambda$-operator internal to EC. Specifically, they assume Partee's (1975) Derived VP Rule, which introduces a $\lambda$-operator at the VP level. (Predicate Abstraction, as in Heim and Kratzer 1998, can be employed to provide the same result.) Their analysis of sloppy identity is illustrated in (3). We will call such variable binding structures, in which all the variables are bound internal to the elided constituent, Internal-binding: 
(3)

\author{
Internal-binding \\ John [AC $\lambda \mathbf{x}$. $\mathbf{x}$ admires $\mathbf{x}$ 's professor]
}

Bill also does $<_{E C} \lambda y$. $y$ admires y's professor $>$

It is easy to see that Internal-binding in (3) allows EC and AC to be semantically identical. The more general prediction that follows from the proposals made by Sag and Williams is that the variable binding structure in (4), which we will call Re-binding, is never allowed.

$$
\begin{aligned}
& \text { Re-binding } \\
& \text { Antecedent Clause: }\left[\ldots\left[\mathrm{XP}_{\mathrm{x}} \ldots[\mathrm{AC} \ldots \mathrm{x} \ldots]\right]\right] \\
& \text { Ellipsis Clause: } \quad\left[\ldots\left[\mathrm{YP}_{\mathrm{y}} \ldots<\mathrm{EC} \ldots \mathrm{y} \ldots>\right]\right]
\end{aligned}
$$

In Re-binding, there are variables that are free inside EC and AC, and there are binders outside $\mathrm{EC}$ and $\mathrm{AC}$ that bind the variables. The prediction that Re-binding is never possible follows from the simple parallelism condition introduced above (together with the assumption mentioned in endnote 1, or (26)), and Sag and Williams support it by a variety of empirical observations. Counter-evidence, however, has been accumulated over the years (Evans 1988, Fiengo and May 1994, Jacobson 1992, Merchant to appear, Schuyler 2001, among others).

We argue that the empirical discrepancy in the past literature results from the fact that Re-binding is allowed, but is constrained. Building on a proposal made by Merchant (to appear), we claim that in Re-binding contexts, ellipsis must target the largest deletable constituent (MaxElide). (See also Fiengo and May 1994:106-7 and Kennedy 2002 for relevant discussion.) The puzzling fact to be discussed is that the effects of MaxElide are observable only in Re-binding environments.

One could accommodate this fact by stipulation, namely, by a direct restriction of MaxElide to the relevant environments. We argue instead that MaxElide applies in those syntactic domains that are relevant for the evaluation of the parallelism condition on ellipsis. As we will see, the relevant syntactic domains have to be relatively big in Re-binding configurations. In other contexts, they can be as small as ECs themselves, thereby leading to the impression that MaxElide is not active.

The difference in the size of the relevant syntactic domains is a direct consequence of a Rooth-type theory of the parallelism condition (Rooth 1992b) when embedded in a system that makes use of variables and variable names. To the extent that our account is successful, it might provide an argument for such a system. 


\section{The Re-binding Puzzle}

\subsection{Evidence for the Sag-Williams Position}

The Sag-Williams parallelism condition is not only conceptually natural, but is empirically supported, as well. The condition entails that sloppy identity is possible only when there is a way to analyze the relevant structure as one of Internal-binding (as in (3)). The Derived VP Rule makes such an analysis possible only when the understood antecedent of the relevant variable is the sister of EC. Sag and Williams claim that this is a good result. In (5b), where the embedded VP is deleted, sloppy identity. is not possible since it cannot result from an Internal-binding structure (in contrast to (5a)).

a. John said Mary hit him, and BILL also did $<\lambda x$. $x$ say Mary hit $x>$.

b. *John said Mary hit him, and BILL also $\lambda x$. $x$ said she did $<\lambda y$. y hit $x>$. (adapted from Sag 1976:131)

Sloppy identity in $(5 \mathrm{~b})$ requires a Re-binding configuration: the variable $\mathrm{x}$ must be free within EC and can only be bound from a matrix position, by the matrix subject or by the $\lambda$-operator introduced by the Derived VP Rule at the matrix VP level. The contrast in (6) makes the same point.

(6) a. John is proud that there are pictures of him there, and BILL is $<\lambda x$. $x$ proud that there are pictures of $x$ there $>$, too.

b. *John is proud that there are pictures of him there, and BILL is $\lambda \mathbf{x} . \mathbf{x}$ proud that there are <pictures of $x$ there>, too. (Williams 1977:122)

The Sag-Williams claim receives further empirical support from the contrast in (7). The unacceptability of (7b) suggests that Re-binding as a consequence of a movement operation is not permitted, as well (Merchant to appear, Sag 1976, Schuyler 2001 and Williams 1977).

(7) a. John knows which prof essor we invited, but he is not allowed to reveal which one $<\lambda x$. we invited $x>$.

b. *John knows which professor we invited, but he is not allowed to reveal which one $\lambda x$. we did $<\lambda y$. y invite $x>$.

\subsection{Evidence Against the Sag-Williams Position}

Contrary to the Sag-Williams position, it has been observed that Re-binding is sometimes allowed in ellipsis. In (8), sloppy identity is acceptable, just like in (5a) 
and (6a), even though, in this case, it cannot result from an Internal-binding structure. (See Jacobson 1992 for relevant observations.)

(8) John argued that Mary hit him, butBILL $\lambda x$. $x$ DENIED that she did $<\lambda y$. y hit $\mathbf{x}>$.

Similarly, Re-binding structures created by movement are also sometimes allowed (Evans 1988, Fiengo and May 1994, among others):

(9) Mary doesn't know who we can invite, but she can tell you who $\lambda x$. we can NOT $<\lambda y$. $y$ invite $x>$.

What is the difference between (5)-(7) and (8)-(9) that might account for the fact that Re-binding is allowed only in the latter? A possibility that suggests itself is that the distinguishing property is the existence of focused material between the re-binder and the re-bound variable (Intervening Focus). In (8), the matrix verb, denied, is focused, and in (9), negation is. One might suggest that this Intervening Focus allows for Re-binding. In (5)-(7), by contrast, there is no Intervening Focus, and Re-binding is impossible. But, why should Intervening Focus be required for Re-binding? We will suggest a possible answer in the next section.

\section{The Generalization}

\subsection{MaxElide}

The constraint in (10), suggested in Fiengo and May (1994), Kennedy (2002) and Merchant (to appear), might be relevant to our concerns.

(10) MaxElide (first pass): Elide the biggest deletable constituent.

In all of the unacceptable Re-binding cases, there is a bigger constituent that could have been deleted. In (7b), repeated here as (11a), the matrix VP could have been deleted, as illustrated in (7a), repeated as (11b). However, the situation is different in the acceptable cases. In these cases, Intervening Focus blocks deletion of a bigger constituent, as illustrated in (11c), where negation is focused. ${ }^{2}$

(11) a. *John knows which professor we invited, but he is not allowed to reveal which one $\lambda x$. we did $\langle\lambda y$. y invite $x\rangle$.

b. John knows which professor we invited, but he is not allowed to reveal which one $\langle\lambda x$. we invited $x>$.

c. Mary doesn't know who we can invite, but she can tell you who $\lambda x$. we can NOT $<\lambda y$. $y$ invite $x>$. 


\subsection{Circumvention of MaxElide}

Unfortunately, there are many counter-examples to MaxElide, as formulated in (10). One such counter-example is (12a), which is acceptable despite the possibility of deleting a bigger constituent, (12b).

(12) a. John said Mary likes Peter. BILL also said she does <like Peter>.

b. John said Mary likes Peter. BILL also did <say Mary likes Peter>.

In response to the contrast between (11a) and (11b), Merchant (to appear) restricts the application of MaxElide to cases in which EC contains an $A^{\prime}$-trace:

(13) MaxElide (adapted from Merchant to appear):

Elide the biggest deletable constituent if EC contains an $\mathrm{A}^{\prime}$-trace.

If MaxElide is to help us capture the Re-binding facts, it has to be further restricted, in order to accommodate cases of sloppy identity such as (5) and (6). The sentences in ( $5 b$ ) and (6b) should be ruled out by MaxElide, just like (11a), due to the fact that deletion of a bigger constituent is possible ((5a) and (6a)). Thus, MaxElide might be modified as follows:

(14) MaxElide (minor modification):

Elide the biggest deletable constituent if EC contains a variable that is free within EC.

However, MaxElide needs to be modified further because the presence of a variable free within EC does not always require ellipsis of the biggest deletable constituent. In (15), EC contains a variable, which is free within EC. Despite this fact, the possibility of deleting the bigger VP in (15a) does not exclude (15b), where the smaller VP is elided, unlike in Re-binding cases (e.g., (5b), (6b) and (11a)).

a. I know which puppy $\lambda x$. you said Mary would adopt $x$ and FRED did $<$ say she would adopt $x>$, too.

b. I know which puppy $\lambda \mathbf{x}$. you said Mary would adopt $\mathrm{x}$ and FRED said she would <adopt $\mathrm{x}>$, too.

The difference between (15) and cases of Re-binding is that in the former, a single element binds the variables which are free within AC and EC (i.e., which puppy in (15)). Configurations in which a single binder of this sort exists, configurations of Co-binding, are schematically represented in (16). The relevant distinction is that in Re-binding, unlike Co-binding, the variables in $\mathrm{EC}$ and $\mathrm{AC}$ are bound by distinct binders, as was illustrated in (4): 
(16)

$$
\begin{aligned}
& \text { Co-binding } \\
& {\left[\ldots\left[\mathrm{XP}_{\mathrm{x}} \ldots[\ldots \mathrm{x} \ldots] \text { and }<\mathrm{EC} \ldots \mathrm{x} \ldots>\right]\right]}
\end{aligned}
$$

The circumvention of MaxElide in Co-binding environments suggests that it is necessary to further restrict MaxElide to Re-binding contexts:

a. MaxElide (final version):

Elide the biggest deletable constituent in Re-binding configurations.

b. Re-binding:

A structure in which $\mathrm{EC}$ dominates a variable that is free within $\mathrm{EC}$ and is bound by a binder YP outside EC, and there is no variable in AC bound by YP.

What remains puzzling is why such a complicated principle should be part of grammar.

\section{The Proposal}

We argue that a complicated principle such as (17) does not exist. What exists, instead, is the simple constraint that prohibits ellipsis of small constituents under all circumstances, i.e., something close to (10). However, we claim that this constraint, MaxElide, applies to particular syntactic constituents - to those constituents that are subject to the parallelism condition on ellipsis. As we will see shortly, these constituents have to be relatively big in Re-binding environments. In other contexts, they can be as small as ECs themselves, thereby leading to the impression that MaxElide is not active.

As mentioned above, the Sag-Williams parallelism condition always applies to EC and $\mathrm{AC}$ and requires that they be semantically identical. In Re-binding, which is represented again in (18), the presence of free variables within these constituents ensures that the condition will not be satisfied.

$$
\begin{aligned}
& \text { Antecedent Clause: }\left[\mathrm{zP} \mathrm{XP}_{\mathrm{x}} \ldots[\ldots[\mathrm{AC} \ldots \mathrm{x} \ldots]]\right] \\
& \text { Ellipsis Clause: } \quad\left[\mathrm{wP}_{\mathrm{P}} \mathrm{YP}_{\mathrm{y}} \ldots[\ldots<\mathrm{EC} \ldots \mathrm{y} \ldots>\text { ] }]\right.
\end{aligned}
$$

Indeed, any constituent in (18) will not be semantically identical to any constituent in the antecedent clause (besides the special case where $[[\mathrm{XP}]]=[[\mathrm{YP}]]$, in which [[ZP]] might be identical to [[WP]]). However, WP, where the variable y is bound, bears a certain relationship to ZP in the antecedent clause, a relationship that various researchers capitalize on. (See in particular Rooth 1992b, and Fiengo and 
May 1994.) Specifically, WP is semantically identical to ZP, modulo focus marked material. In Rooth's (1992a, b, 1996) terms, ZP is a member of the focus value of WP. Rooth (1 992b) claims that this relationship, which holds between constituents bigger than EC and AC, licenses ellipsis of EC in (18). What is going to be crucial is that this relationship, which holds between WP and ZP, does not hold between any constituent smaller than WP and any corresponding constituent in the antecedent clause. A condition on ellipsis along the lines of Rooth's (1992b) is formulated below:

(19) For ellipsis of EC to be licensed, there must exist a constituent, which reflexively dominates $\mathrm{EC}$, and satisfies the parallelism condition in (20). ${ }^{3}$

We will call such a constituent a Parallelism Domain, or a PD:

(20) Parallelism

PD satisfies the parallelism condition if PD is semantically identical to another constituent $A C$, modulo focus marked constituents.

PD is semantically identical to AC modulo focus marked constituents, if there is a focus alternative to $\mathrm{PD}, \mathrm{PD}_{\mathrm{Alt}}$, such that for every assignment function, $g,\left[\left[\mathrm{PD}_{\mathrm{Alt}}\right]\right]^{\mathrm{g}}=[[\mathrm{AC}]]^{\mathrm{g}}$.

$\mathrm{PD}_{\text {Alt }}$ is an alternative to $\mathrm{PD}$ if $\mathrm{PD}_{\mathrm{Alt}}$ can be derived from $\mathrm{PD}$ by replacing focus marked constituents with their alternatives. ${ }^{4}$

Unlike the Sag-Williams parallelism condition, the size of constituents to which the parallelism condition applies can vary in a Rooth-type theory. Furthermore, the range of possible variation depends on the presence of a re-bound variable in EC and on the position from which it is bound. If there is such a variable, the PD must be a constituent that dominates the re-binder, i.e., it must be bigger than EC. However, if there is no such variable, EC itself can be taken as the PD. We capitalize on this difference to capture the fact that MaxElide effects are observable only in Re-binding environments. Specifically, we propose that MaxElide, as defined in (10), applies in Parallelism Domains:

MaxElide (our proposal):

Elide the biggest deletable constituent reflexively dominated by PD.

As mentioned above, if there is no re-bound variable involved, EC itself can be a PD. Thus, MaxElide is trivially satisfied both in (22a) and in (22b), which have previously been taken to involve MaxElide circumvention. Notice that there is no bigger deletable constituent within the PD since in these cases, PD $=E C$ : 
(22) a. John said Mary likes Peter. BILL also said she does [PD $<_{E C}$ like Peter $>$ ].

b. John said Mary likes Peter. BILL also did [PD $<_{E C}$ say Mary likes Peter $>$ ].

In Re-binding contexts repeated here as (23a), the embedded CP is the smallest possible PD for reasons stated more generally with respect to (18).

a. *John knows which professor we invited, but he is not allowed to reveal [PD which one $e_{1}$ we did $<_{E C}$ invite $t_{1}>$ ].

b. John knows which professor we invited, but he is not allowed to reveal [PD which one ${ }_{1}<_{E C}$ we invited $t_{1}>$ ].

c. Mary doesn't know who we can invite, but she can tell you [PD who we can NOT $<$ EC invite $t_{1}>$ ].

MaxElide demands deletion of the biggest deletable constituent within this domain, which is the embedded TP. ${ }^{5}$ Thus, VP-ellipsis in (23a) is ruled out by MaxElide because it involves deletion of a smaller constituent than the embedded TP. As expected, the sentence in (23b) is acceptable since the embedded TP is deleted. ${ }^{6}$ Like in (23a) and (23b), the embedded CP is the smallest PD in (23c) where Intervening Focus is involved. Since focus marked material cannot be deleted, the elided VP is the biggest deletable constituent in this sentence.

Consider now instances of Co-binding, repeated here in (24).

a. I know which puppy $y_{1}$ you said Mary would [AC adopt $t_{1}$ ] and FRED said she would [PD $<_{E C}$ adopt $\mathrm{t}_{1}>$ ], too.

b. I know which puppy you [AC said Mary would adopt $t_{1}$ ] and FRED did [PD $<$ EC say she would adopt $\mathrm{t}_{1}>$ ], too.

In Co-binding, just like Re-binding, variables are free within EC and AC. However, in Co-binding, all variables are bound by a single binder. Thus, they share the same variable name. For this reason, the elided embedded VP can be semantically identical to AC in (24a) and, hence, can serve as a Parallelism Domain. Therefore, MaxElide is obeyed in (24a), just like in (22a). The possibility of deleting the bigger VP in (24b) does not preclude (24a) since in the latter, MaxElide applies to the embedded VP, and within this constituent, deletion is indeed maximal. ${ }^{7}$

The identity of variable names plays a crucial role in the account of the Co-binding facts. Given this, one might suggest that a structure analogous to Co-binding could be postulated in Re-binding by assigning the same variable name to both the variables within AC and EC, as illustrated in (25). In this representation, $\mathrm{EC}$ and $\mathrm{AC}$ would be semantically identical because they involve variables with the same name, like in Co-binding. Consequently, the embedded VP would be a possible target for deletion, contrary to fact: 
*John knows which professor 1 we [invited $t_{1}$ ], but he is not allowed to reveal which one $e_{1}$ we did $\left[\mathrm{PD}<\mathrm{EC}\right.$ invite $\left.\mathrm{t}_{1}>\right]$.

For this and other reasons (Heim 1997), we are committed, like Sag and Williams, to the claim that identical variable names cannot be assigned to variables bound by distinct binders, as stated in (26) (cf., Kennedy 2004). Thus, there is no way to regard EC itself as a PD in Re-binding configurations:

No Meaningless Coindexation

If an LF contains an occurrence of a variable $v$ that is bound by a node $\alpha$, then all occurrences of $v$ in this LF must be bound by the same node $\alpha$.

(Heim 1997:202; see also Sag 1976:180)

\section{Further Evidence}

\subsection{Re-binding as a Consequence of Covert Movement}

In the previous sections, we have discussed the effects of MaxElide when Re-binding results from overt $w h$-movement (i.e., the contrast between (23a) and (23b)). However, we should expect to find the same effects when a Re-binding configuration is created by other types of movement. We will see evidence that this expectation is borne out in the case of covert movement, more specifically, Quantifier Raising $(\mathrm{QR})$.

To set the stage, we first present a well-known argument that $\mathrm{QR}$ can create a Re-binding configuration. Hirschbühler (1982) discovered that an object can take wide scope over the subject in VP-ellipsis. This is illustrated in (27).

(27) A doctor treated every patient. A NURSE did <treat every patient $>$, too. $(\exists>\forall)(\forall>\exists)$

(Hirschbühler 1982)

Object wide scope is derived from the representations in (28) in which the object quantifier occupies a structurally higher position than the subject.

Antecedent Clause: [every patient $t_{1}\left[a\right.$ doctor [treated $\left.t_{1}\right]$ ]]

Ellipsis Clause: [PD every patient ${ }_{2}\left[\mathrm{a}\right.$ nurse $<_{E C}$ treated $t_{2}>$ ]]

These representations involve variables that are bound by two distinct constituents (the object quantifiers). If the object of the elided verb is outside EC, (27) involves Re-binding. ${ }^{8}$ This means that the Parallelism Domain must include the re-binder, as depicted in (28). Since the subject, a nurse, in the ellipsis clause is focus marked 
in (27), the elided VP is the biggest deletable constituent. Thus, the Re-binding configuration that results from object wide scope is permitted in (27). ${ }^{9}$

Given this discussion, we expect to find cases where object wide scope is ruled out by MaxElide. More specifically, we correctly predict object wide scope to be impossible in (29a) (observed by Williams 2003).

(29) a. At least one doctor tried to get me to arrest every patient, and at least one NURSE tried to get me to <arrest every patient>, as well. $(\exists>\forall) *(\forall>\exists)$

b. At least one doctor tried to get me to arrest every patient, and at least one NURSE did <try to get me to arrest every patient>, as well. $(\exists>\forall) ?(\forall>\exists)$

(Williams 2003)

As illustrated in (30), which is the representation of object wide scope for the ellipsis clause in (29a) and (29b), there is only one possible PD, namely, the entire ellipsis clause. Since there is a possibility of deleting the bigger VP, (29b), ellipsis of the smaller VP is impossible in $(29 a):^{10}$

(30) [PD every patient $t_{1}$ [at least one nurse [VP tried to get me to [vP arrest $\left.\left.t_{1}\right]\right]$ ]

Williams' (2003) interpretation of the facts is different. On the basis of the unavailability of object wide scope in (29a), he concludes that Re-binding is in general impossible. ${ }^{11}$ However, our alternative is corroborated by the fact that object wide scope is permitted even when the smaller VP is elided as long as Intervening Focus prevents ellipsis of the matrix VP, (31). We take this fact as further evidence for our claim that Re-binding is allowed, but is constrained by MaxElide: ${ }^{12}$

(31) A doctor tried to arrest every patient, and a NURSE MANAGED to <arrest every patient $>$. $(\exists>\forall)(\forall>\exists)$

\subsection{The Position of Intervening Focus}

We have argued that Intervening Focus is required for Re-binding. If there is Intervening Focus, MaxElide is satisfied as a consequence of the fact that ellipsis of any constituent dominating the Intervening Focus is blocked. However, it is crucial to notice that for Re-binding to be allowed, the biggest deletable constituent up to Intervening Focus has to be elided. Even in cases where Intervening Focus is involved, Re-binding should be impossible if the biggest deletable constituent is not elided. 
This is indeed what we find in (32). Here, negation, not, is the relevant focus marked material. In (32), there are two deletable constituents, namely, the intermediate VP headed by agree and the most embedded VP headed by adopt. Ellipsis of the most embedded VP in (32a) is excluded by MaxElide, due to the possibility of deleting the intermediate VP demonstrated in (32b).

a. *I don't know which puppy you should agree to adopt, but I know [PD which one 1 you should NOT [VP agree to $<_{\mathrm{EC}}$ adopt $\mathrm{t}_{\mathrm{l}}>$ ]].

b. I don't know which puppy you should agree to adopt, but I know [PD which one 1 you should NOT $<_{\text {EC }}$ agree to adopt $t_{1}>$ ].

As illustrated in (33), deletion of the most embedded VP is permitted if Intervening Focus (refuse in (33)) occupies a structurally low position so that its existence prohibits ellipsis of any constituent bigger than EC.

(33) I don't know which puppy you should agree to adopt, but I know [PD which one $_{1}$ you should REFUSE to $<_{\text {EC }}$ adopt $t_{1}>$ ].

\subsection{Syntactic Licensing Conditions}

As we saw above, the presence of focus marked material diminishes the number of candidates for ellipsis. (All dominating constituents cannot be deleted.) We suggest that certain syntactic licensing conditions on ellipsis have the same effect. As illustrated in (34a), EC cannot be a constituent headed by the perfective auxiliary, have.
a. *Sally might have eaten rutabagas, but Holly should NOT < have eaten rutabagas>.
(Johnson 2001:442)
b. Sally might have eaten rutabagas, but Holly should NOT have <eaten rutabagas $>$.

The same is also true in Re-binding, (35a). If the constituent headed by have could have counted as a candidate that is relevant for the evaluation of MaxElide, (35b) would have been ruled out since a smaller constituent is elided. Thus, the facts in (35) suggest that MaxElide is a principle that demands deletion of the biggest constituent that obeys other conditions of grammar, among them, the syntactic licensing conditions on ellipsis and the requirement that focus be overtly realized.
a. *I know which dish Sally should have eaten, but I don't know [PD which one $_{1}$ she should NOT $<$ have eaten $\mathrm{t}_{1}>$ ].
b. I know which dish Sally should have eaten, but I don't know [PD which one ${ }_{1}$ she should NOT have <eaten $\mathrm{t}_{1}>$ ].




\section{Evidence for Variables}

In Re-binding, the Parallelism Domain must dominate the re-binder. Thus, the Parallelism Domain is necessarily bigger than EC in this configuration. In contrast, we have suggested that EC itself can be a Parallelism Domain in other contexts. In order to differentiate Re-binding from other cases, we have made crucial use of variables and variable names.

However, variables and variable names do not play any role in variable-free semantics (Jacobson 1992, 1999, among others). Specifically, a pronoun does not make any contribution to the meaning of an elided VP in sloppy identity construction such as (36). ${ }^{13}$

Therefore, the meaning of the embedded VP in (36) is the same as the meaning of the verb hit. Under this assumption, EC itself could be taken as the PD in (36) (because it is semantically identical to the embedded VP in the antecedent clause). If this is a possible option, MaxElide would be satisfied in (36) and the sentence would be acceptable, contrary to fact.

*John said Mary [AC hit him], and BILL also said she did [PD $<_{\mathrm{EC}}$ hit $>$ ].

Is there a formulation of MaxElide that will have the desired consequences within variable-free semantics? We do not know. (See Jacobson 2004 for a potentially relevant proposal.) If the answer is negative, we can take our results to argue in favor of systems that postulate variables and variable names.

\section{Further Issues}

\subsection{Focused Traces}

Schuyler (2001) provides potential counter-examples to MaxElide. The sentences in (37) are problematic because they are acceptable even though there is a possibility of deleting a bigger constituent than EC (i.e., the TP in (37a) and the matrix VP in (37b)).
a. ?I don't know which puppy you should adopt, but I know which
KITTEN $N_{1}$ [TP you should <adopt $\mathrm{t}_{1}>$ ].
b. The blue papers I think Pete signed, and the GREEN ones I I vP think he did $<$ sign $t_{1}>$ ], too.
(Schuyler 2001:8, 13)

Two properties are common to the potentially problematic cases: the re-binder is a phrase that has moved out of EC, and the re-binder bears contrastive focus. These properties seem crucial, as suggested by facts already discussed. The 
ungrammatical sentences in (38a) and (38b) lack the first and the second property, respectively.

(38) a. ${ }^{*} J_{o h n_{1}}$ said Mary hit him 1 , and BILL 2 also said she did $<$ hit him $2>$.

b. *John knows which professor we invited, but he is not allowed to reveal which one o $_{1}$ wid $<_{V P}$ invite $t_{1}>$.

We suggest, following Sauerland (1998), among others, that if an element in a head of a movement chain is focused (which kitten in (37a) and the green ones in (37b)), focus is optionally present within the trace. Together with the assumption that movement takes place successive-cyclically (e.g., moved phrases adjoin to every VP and $\mathrm{CP}$ ), (37b) could involve the following representation:

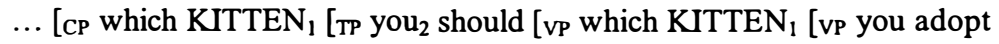
which kitten $\left._{1}\right]$ ]]

Ellipsis of a larger constituent than EC is prohibited in (37) because the focused trace in the VP-adjoined position functions as Intervening Focus. ${ }^{14}$

\subsection{The $A / A$ '-distinction}

Lasnik (2001) suggests that when a subject is extracted by wh-movement, the possibility of deleting TP does not preclude VP-ellipsis, as illustrated in (40). Based on this fact, Merchant (to appear) claims that MaxElide applies only to cases in which EC involves A'-traces, as was stated in (13):

(40) a. Someone solved the problem.

b. 1. Who $<\left[\operatorname{TP}^{\mathrm{A}^{\mathrm{A}}}\left[\mathrm{VP}_{\mathrm{P}} \mathrm{t}^{\mathrm{A}}\right.\right.$ solved the problem $\left.]\right]>$ ?

2. Who did $<\left[\mathrm{vp}^{\mathrm{A}}\right.$ solve the problem $]>$ ?

Contrary to Merchant, our formulation of MaxElide in (21) predicts (40b2) to be unacceptable if the subject $w h$-phrase is in Spec, CP: the PD would be the entire CP. If, however, we assume that wh-movement of a subject to Spec,CP is optional, there would be a way to regard (40) as a non-Re-binding case. If the subject moves to Spec, CP, (40bl) is derived. If $w h$-movement does not take place, the derivation ends up with VP-ellipsis, (40b2).

Raising constructions like (41) seem to be more appropriate for investigating whether there is an $\mathrm{A} / \mathrm{A}^{\prime}$ distinction in the application of MaxElide. So far, we found variation in the judgments of these sentences. If it tums out that $(41 \mathrm{~b})$ is acceptable, it will suggest that movement introduces a $\lambda$-operator and $\lambda$-predicates could be taken as PDs. Given these assumptions, successive-cyclic A-movement allows us to analyze (41) with an Internal-binding structure. ${ }^{15}$ 
(41) a. I know that John is likely to win the election, but I am not allowed to reveal that he is <likely to win the election>.

b. I know that John is likely to win the election, but I am not allowed to reveal that he is likely to <win the election>.

\subsection{Jacobson's Problem}

Pauline Jacobson (personal communication) points out that the effects of MaxElide are observable also in cases where Re-binding is not involved. The sentence in (42a) is unacceptable and this might be attributed to the possibility of deleting a bigger constituent than EC, as illustrated in (42b). Notice that (42a) can be analyzed as an Internal-binding structure since all the variables are bound internal to the elided constituent. Since the Parallelism Domain could have been as small as EC itself in (42a), MaxElide should have been satisfied.
a. *John loves every woman who skis, and BILL also loves every woman who does $<\lambda \mathbf{x}$. $x$ ski $>$.
b. John loves every woman who skis, and BILL also does $<\lambda x$. $x$ love every woman who skis $>$. (Pauline Jacobson, personal communication)

We do not have a solution to Jacobson's puzzle. We hope that the correct solution lies in constraints on the choice of Parallelism Domains. Since a complex NP intervenes between two Parallelism Domains, PD1 and PD2, as illustrated in (43), we would like to explore constraints that refer to the presence of islands. However, detailed investigation of this issue needs to be left for future research:

[PD1 BILL also love [island every woman [PD2 who skis]]]

\section{Conclusion}

We have claimed that MaxElide is a constraint that forces deletion of the biggest deletable constituent under all circumstances. We also claimed that this constraint applies to those constituents which are subject to the parallelism condition (Parallelism Domains). Parallelism Domains must be bigger than ECs in Re-binding contexts, but in other contexts, they can be the ECs themselves. This explains the fact that the effects of MaxElide are observable only in Re-binding contexts. The difference in the size of Parallelism Domains is a corollary of context-sensitive parallelism conditions (such as Rooth's 1992b) in a system that postulates variables and variable names. Thus, we have suggested that our results can be taken as evidence for such systems. 


\section{Endnotes}

* We have presented parts of this paper in GLOW 2005, SALT XV, and the syntax-semantics reading group at MIT. We would like to thank the audience in these talks for their comments. We are grateful to Pauline Jacobson and Uli Sauerland for invaluable discussion and their questions. All remaining errors and inadequacies are our own.

${ }^{1}$ Specifically, if we assume, following Heim (1997), that the variables in (2) must bear different names, the Sag-Williams parallelism condition will not be satisfied: EC will not be semantically identical to AC (under every assignment function). Heim's assumption is stated in (26).

${ }^{2}$ The effects of MaxElide are also observable in the sentences in (i), which do not involve (overt) movement in the antecedent clause.

(i) a. *I know we invited someone, but I can't remember WHO $\lambda x$. we did $<\lambda y$. $y$ invite $x>$.

b. I know we invited someone, but I can't remember WHO $<\lambda x$. we invited $\mathrm{x}>$.

c. It's clear that they could invite someone, but I don't know who $\lambda \mathbf{x}$. they ever WOULD $<\lambda y$. $y$ invite $x>$.

(Schuyler 2001:7)

If Re-binding is involved in these cases (as is widely assumed), they could be treated on a par with (11). See Fox and Lasnik (2003) and Merchant (to appear) for relevant discussion of these cases.

${ }^{3} \mathrm{XP}$ reflexively dominates $\mathrm{YP}$ if $\mathrm{XP}$ dominates $\mathrm{YP}$ or $\mathrm{XP}=\mathrm{YP}$.

${ }^{4}$ Parallelism in (20) is satisfied if AC and PD are semantically identical. This is different from Rooth's (1992a, 1996) Focus Interpretation Principle. We hope that our modification will not be problematic for other areas where the theory of focus interpretation is implemented.

${ }^{5}$ The CP cannot be deleted for syntactic reasons. See 5.3 for relevant discussion.

${ }^{6}$ In our analysis, the choice of PD determines the size of a constituent to be deleted. Sauerland $(1998,1999)$ claims that the choice of PD determines how much of a constituent should be focused.

${ }^{7}$ One attractive implementation of our idea relies on the assumption that deletion can apply at the course of the derivation. In the non-Re-binding cases, a constituent could be elided before a bigger deletable constituent is created. In the Re-binding context, deletion cannot apply until a re-binder is introduced into the derivation, since the parallelism condition is not met before that stage of the derivation.

${ }^{8}$ For theory internal reasons (consistency with the Sag-Williams theory) Hirschbühler suggests a Co-binding analysis of object wide scope, in which an object quantifier (e.g., every patient in (27)) undergoes across-the-board movement to a position structurally higher than the subjects of the two clauses. However, he presents very strong counter-evidence. 
${ }^{9}$ Sag (1976) and Williams (1977) claim that object wide scope is impossible in ellipsis on the basis of sentences like (i). They take this fact as further evidence for their position that Re-binding is never allowed:

$$
\begin{aligned}
& \text { A doctor treated every patient. MARY did <treat every patient>, too. } \\
& (\exists>\forall) *(\forall>\exists)
\end{aligned}
$$

The contrast between (i) and (27) suggests that Re-binding as a consequence of covert movement is only allowed under certain circumstances. See Fox (1995) and Tomioka (1997), among others.

${ }^{10}$ The availability of subject wide scope in (29a) follows straightforwardly. Since the object quantifier adjoins to the most embedded VP by QR in this case, subject wide scope does not involve Re-binding. Thus, the most embedded VP (or TP, see endnote 15) can be chosen as a PD and be targeted for deletion without violating MaxElide.

${ }^{11}$ More specifically, he claims that in (27), the subject quantifier can undergo reconstruction into a VP-internal position, which is below the QRed object. Consequently, there is a way to analyze object wide scope with an Internal-binding structure. In contrast, a control predicate, try, is used in the matrix position in (29), which prohibits a subject from reconstructing into the complement clause.

12 There is a debate as to whether antecedent-contained deletion (ACD) involves Re-binding. (See Heim 1997 for discussion.) Sentences that bear on this debate in the present context would be ones like (i). The interpretation relevant for our purposes is object wide scope:

(i) a. At least one student tried to solve every problem that Prof. SMITH tried to <solve>.

b. At least one student tried to solve every problem that Prof. SMITH did $<$ try to solve $>$.

If the effects of Re-binding are observed in ACD (i.e., if (ia) is degraded under the relevant interpretation), Re-binding analyses would be supported. Since the sentences are difficult to judge, we are unable to say anything conclusive at the present point.

${ }^{13}$ If movement traces were postulated within this framework, they would also be semantically vacuous.

${ }^{14} \mathrm{We}$ suggest that focus marking on traces is optional. Thus, the trace in the complement of VP does not have to be focused, and the VP can be deleted. We also need to say something about the way traces are interpreted. See Fox $(2000,2002)$ and Sauerland (1998, 2004).

${ }^{15}$ Given the assumptions mentioned above, the unacceptable sentence in (ia) where an object undergoes $w h$-movement would involve the representation in (ib). 
(i) a. *John knows which professor we invited, but he is not allowed to reveal which one $e_{1}$ we did $<$ invite $\mathrm{t}_{1}>$.

b. $\quad \ldots[\mathrm{CP}$ which one [PD $\lambda x$. [TP we $\lambda y$. [VP $x \lambda z$. [VP y invite $z]]]]]$

In (ib), the $\lambda$-predicate introduced at the TP level $(\lambda x)$ is the smallest possible Parallelism Domain, due to the presence of traces of $w h$-movement ( $\mathrm{x}$ and $\mathrm{z}$ ) and A-movement of the subject (y). Thus, (ia) is once again ruled out by MaxElide.

\section{References}

Evans, Frederic: 1988, 'Binding into Anaphoric Verb Phrases', in J. Powers and K. de Jong (eds.) Proceedings of the Fifth Eastern States Conference on Linguistics, 122-129, The Ohio State University.

Fiengo, Robert and Robert May: 1994, Indices and Identity, MIT Press, Cambridge, Mass.

Fox, Danny: 1995, 'Economy and Scope', Natural Language Semantics 3, 283-341.

Fox, Danny: 2000, Economy and Semantic Interpretation, MIT Press, Cambridge, Mass.

Fox, Danny: 2002, 'Antecedent-Contained Deletion and the Copy Theory of Movement', Linguistic Inquiry 33, 63-96.

Fox, Danny and Howard Lasnik: 2003, 'Successive-cyclic Movement and Island Repair: The Difference between Sluicing and VP-ellipsis', Linguistic Inquiry 34, 143-154.

Heim, Irene: 1997, 'Predicates or Formulas? Evidence from Ellipsis', in A. Lawson (ed.) Proceedings of SALT VII, 197-221, CLC Publications, Cornell University.

Heim, Irene and Angelika Kratzer: 1998, Semantics in Generative Grammar, Blackwell Publishers.

Hirschbühler, Paul: 1982, 'VP Deletion and Across-the-board Quantifier Scope', in J. Puste jovsky and P. Sells (eds.) Proceedings of NELS 12, 132-139, GLSA Publications, University of Massachusetts, Amherst.

Jacobson, Pauline: 1992, 'Antecedent Contained Deletion in a Variable-free Semantics', in C. Barker and D. Dowty (eds.) Proceedings of SALT II, 193-213, The Ohio State University.

Jacobson, Pauline: 1999, 'Towards a Variable-free Semantics', Linguistics and Philosophy 22, 117-184.

Jacobson, Pauline: 2004, 'Kennedy's Puzzle: What I'm Named or Who I am?', in R. B. Young (ed.) Proceedings of SALT XIV, 145-162, CLC Publications, Cornell University. 
Johnson, Kyle: 2001, 'What VP Ellipsis Can Do, and What It Can't, but Not Why', in M. Baltin and C. Collins (eds.) The Handbook of Contemporary Syntactic Theory, 439-479, Blackwell Publishers.

Kennedy, Christopher: 2002, 'Comparative Deletion and Optimality in Syntax', Natural Language and Linguistic Theory 20, 553-621.

Kennedy, Christopher: 2004, 'Argument Contained Ellipsis Revisited', Ms., Northwestern University.

Lasnik, Howard: 2001, 'When Can You Save a Structure by Destroying It?', in M. Kim and U. Strauss (eds.) Proceedings of NELS 31, 301-320, GLSA Publications, University of Massachusetts, Amherst.

Merchant, Jason: To appear, 'Variable Island Repair under Ellipsis', to appear in K. Johnson (ed.) Topics in Ellipsis, Oxford University Press.

Partee, Barbara: 1975, 'Montague Grammar and the Transformational Grammar', Linguistic Inquiry 4, 203-300.

Rooth, Mats: 1992a, 'A Theory of Focus Interpretation', Natural Language Semantics 1, 75-116.

Rooth, Mats: 1992b, 'Ellipsis Redundancy and Reduction Redundancy', in S. Berman and A. Hestvik (eds.) Proceedings of the Stuttgart Ellipsis Workshop, Report Series, Sprachtheoretische Grundlagen für die Computerlinguistik, no 29, SFB 340, 1-26, IBM Heidelberg.

Rooth, Mats: 1996, 'Focus', in S. Lappin (ed.) The Handbook of Contemporary Semantic Theory, 271-297, Blackwell Publishers.

Sag, Ivan: 1976, Deletion and Logical Form, Doctoral dissertation, MIT, Cambridge, Mass.

Sauerland, Uli: 1998, The Meaning of Chains, Doctoral dissertation, MIT, Cambridge, Mass.

Sauerland, Uli: 1999, 'Why Variables?' in P. Tamanji, M. Hirotani, and N. Hall (eds.) Proceedings of NELS 29, 323-337, GLSA Publications, University of Massachusetts, Amherst.

Sauerland, Uli: 2004, 'The Interpretation of Traces', Natural Language Semantics 12, 63-127.

Schuyler, Tamara: 2001, 'Wh-movement out of the Site of VP-ellipsis', in S. M. Bhloscaidh (ed.) Syntax and Semantics at Santa Cruz 3, 1-20, University of California, Santa Cruz.

Tomioka, Satoshi: 1997, Focusing Effects and NP Interpretation in VP Ellipsis, Doctoral dissertation, University of Massachusetts, Amherst, Mass.

Williams, Edwin: 1977, 'Discourse and Logical Form', Linguistic Inquiry 8, 101-139.

Williams, Edwin: 2003, 'Parallelism vs. Identity in Ellipsis', Talk presented at Colloquia at MIT. 